\title{
REVIEW
}

\section{Osteochondroma Pathogenesis}

\section{Mouse Models and Mechanistic Insights into Interactions with Retinoid Signaling}

\author{
Sonia Arely Garcia, Vincent Y. Ng, Masahiro Iwamoto, PhD, DDS, and Motomi Enomoto-Iwamoto, PhD, DDS
}

From the Department of Orthopaedics, University of Maryland School of Medicine, Baltimore, Maryland

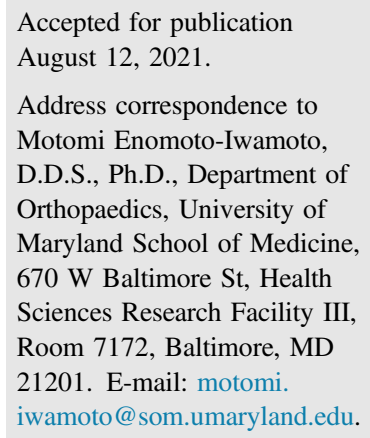

Osteochondromas are cartilage-capped tumors that arise near growing physes and are the most common benign bone tumor in children. Osteochondromas can lead to skeletal deformity, pain, loss of motion, and neurovascular compression. Currently, surgery is the only available treatment for symptomatic osteochondromas. Osteochondroma mouse models have been developed to understand the pathology and the origin of osteochondromas and develop therapeutic drugs. Several cartilage regulatory pathways have been implicated in the development of osteochondromas, such as bone morphogenetic protein, hedgehog, and WNT/ $\beta$-catenin signaling. Retinoic acid receptor- $\gamma$ is an important regulator of endochondral bone formation. Selective agonists for retinoic acid receptor- $\gamma$, such as palovarotene, have been investigated as drugs for inhibition of ectopic endochondral ossification, including osteochondromas. This review discusses the signaling pathways involved in osteochondroma pathogenesis and their possible interactions with the retinoid pathway. (Am J Pathol 2021, 191: 2042-2051; https://doi.org/10.1016/j.ajpath.2021.08.003)
Osteochondromas or exostoses are benign cartilage tumors typically diagnosed during early childhood or puberty. Forty percent of osteochondromas are diagnosed before patients reach 10 years of age. ${ }^{1}$ Osteochondromas can occur as a solitary osteochondroma or multiple osteochondromas as part of a syndrome known as hereditary multiple exostosis (HME). ${ }^{1,2}$ These cartilage-capped protrusions grow away from the physis, creating an outgrowth with a pedunculated or sessile shape. ${ }^{1,2}$

Patients severely affected by HME often have short stature and limb deformities, including limb length inequalities. $^{3}$ Osteochondromas can cause irritation of the overlying soft tissues, neurovascular impingement, and abnormal development of adjacent joints. ${ }^{1-3}$ Plain radiography and axial imaging with either magnetic resonance imaging or computed tomography are used to differentiate osteochondromas from other bone lesions. Symptomatic osteochondromas are typically treated with surgical excision. $^{1,3}$ Depending on the location of the osteochondroma, surgery can vary from simple to very complex. Nearby critical structures can pose a significant risk for permanent injury. ${ }^{3}$ If the entire cartilage cap is not removed, osteochondromas can recur. Malignant transformation to secondary chondrosarcomas, particularly of large osteochondromas or of those in the context of severe HME, is a relatively rare but well-described phenomenon. The option for nonsurgical treatment of small osteochondromas or for osteochondromas within anatomically challenging locations would reduce the need for future aggressive operations, avoid growth abnormalities, and prevent malignant transformation.

Retinoic acid receptor (RAR)- $\gamma$ is an important regulator of cartilage and bone development and growth. ${ }^{4}$ A previous study demonstrated that RAR- $\gamma$ stimulation by RAR- $\gamma$ agonist inhibited experimental heterotopic ossification,

Supported by NIH grant R01AR073181 (M.E.I.).

Disclosures: None declared. 
which is characterized as an excess endochondral bone formation that accumulates within connective and muscle tissues. ${ }^{5}$ In addition, independent studies have reported RAR- $\gamma$ inhibitory action using different heterotopic ossification animal models. ${ }^{6,7}$ More importantly, palovarotene, a synthetic agonist for RAR- $\gamma$, strongly inhibits osteochondroma formation in the mouse model. ${ }^{8}$ These preclinical studies led to a clinical trial of pediatric patients to evaluate the efficacy and safety of palovarotene for the systemic treatment of HME and the prevention of disease progression (https://clinicaltrials. gov; trial number NCT03442985). Elucidation of the precise molecular and cellular mechanism of RAR- $\gamma$ agonists on osteochondromas and ectopic endochondral formation will provide useful information to develop effective pharmacologic therapies.

This review provides an overview of available murine models, useful for basic and translational osteochondroma studies. Signaling pathways that have been demonstrated to be implicated in pathogenesis of osteochondromas are discussed with the possible interaction of these pathways with RAR- $\gamma$ signaling in which pharmacologic activation would result in inhibition of osteochondromas.

\section{Molecular Pathology of Multiple Osteochondromas}

Multiple formation of osteochondromas is a characteristic of HME, which is a pediatric, autosomal-dominant disorder with reported incidences of approximately 1 per 50,000 individuals worldwide. ${ }^{1-3}$ Patients with HME have autosomal dominant functional mutations in the EXT1 and/or EXT2 genes, triggering the development of multiple osteochondromas. ${ }^{9,10}$ EXT mutations include frame shift, missense, and splice-site mutations. ${ }^{11}$ The human EXT1 and EXT2 genes encode endoplasmic reticulum-localized type II transmembrane glycoproteins that are tightly associated with glycosyltransferase activities. ${ }^{12}$ Exostosin 1 (EXT1) and exostosin 2 (EXT2) glycosyltransferases form a stable hetero-oligomeric complex that accumulates in the Golgi apparatus and are involved in heparan sulfate proteoglycan (HSPG) biosynthesis. ${ }^{12}$

HSPGs consist of extracellular core proteins in which a tetrasaccharide linker is synthesized on conserved serine residues of the core protein of HSPGs. EXT1 and EXT2 allow the elongation of heparan sulfate (HS) chains by sequentially adding alternating units of $\mathrm{N}$-acetylglucosamine and glucuronic acid. As HS chains are created, deacetylation, sulfation, and epimerization occur. ${ }^{13}$ Four HSPG isoform families have been identified: syndecan, glypican, perlecan, and CD44. ${ }^{12}$ Glypicans and syndecans that carry long HS chains specifically bind and interact with signaling proteins, plasma proteins, and growth factors. Therefore, these HSPGs affect a variety of neighboring cell responses, such as cell differentiation, cell-to-cell interaction, receptor trafficking, and control tissue morphogenesis and gradients of growth factors, such as fibroblast growth factors, bone morphogenetic proteins (BMPs), hedgehogs (HHs), and Wnts in the extracellular matrix. ${ }^{13}$ The pathogenesis of osteochondroma development has not been clearly elucidated; however, HS synthesis deficiency most likely underlies the molecular mechanism of osteochondroma formation. ${ }^{12}$

\section{Current Mouse Models of Osteochondromas}

Because osteochondromas are located close to growth plate, osteochondroma may presumably arise from growth plate or neighboring connective mesenchymal cells potentially caused by misregulation of chondrogenesis and/or endochondral ossification during skeletal development and growth. Several mouse models have been generated by systemic or cartilage- or perichondrium-specific inactivation of $E x t 1$ and/or Ext2, and have been used to study pathology and pharmacotherapy of osteochondromas (Table $1^{14-20}$ ).

\section{Col2-rTA-Cre;Ext1e2neoflox/e2neoflox with Doxycycline}

The dominant inheritance of HME might indicate that heterozygous mutations in the EXT1 or EXT2 gene would result in systemic reduction of HS levels, leading to osteochondroma formation. However, haploinsufficiency might not always trigger osteochondroma formation in a number of patients with HME. ${ }^{21}$ A loss of heterozygosity model has been proposed to explain the molecular pathogenesis of multiple osteochondromas. The EXTI single-allele mutation experiences a second somatic mutation in a normal EXT allele in osteochondromas occurring at multiple lesions in patients with HME. ${ }^{22}$ Jones et $\mathrm{al}^{14}$ developed the transgenic mice that harbor an inducible loss-of-function allele of Ext 1 (Extle2neofl or Extle2fl) in which exon 2 was flnked by head-to-head loxP sites and determined the necessity for loss of heterozygosity in osteochondroma formation. They demonstrated that clones of proliferating chondrocytes in the osteochondroma tissue are homozygous for the inverted Extl allele and are negative for HS staining. Thus, this study indicates that loss of heterozygosity is critical for osteochondroma formation.

\section{Col2CreER;Ext1fl/fl without Tamoxifen}

Matsumoto et $\mathrm{al}^{15}$ created conditional compound mouse mutants bearing a standard floxed Extl gene $($ Extlfl/fl) and Col2CreERT transgene that directs tamoxifen-inducible Cre recombinase expression under the Col2al promoter/enhancer control. Col2-CreERT;Extlfl/fl mice developed multiple osteochondromas in long bones and other HME-associated skeletal defects (short stature, bowing deformity of the forearm, subluxation or dislocation of the radius, and scoliosis) without tamoxifen treatment caused by a low level of stochastic leakiness of the Cre recombinase activity. 
Table 1 Summary of Mouse Multiple Osteochondromas Models

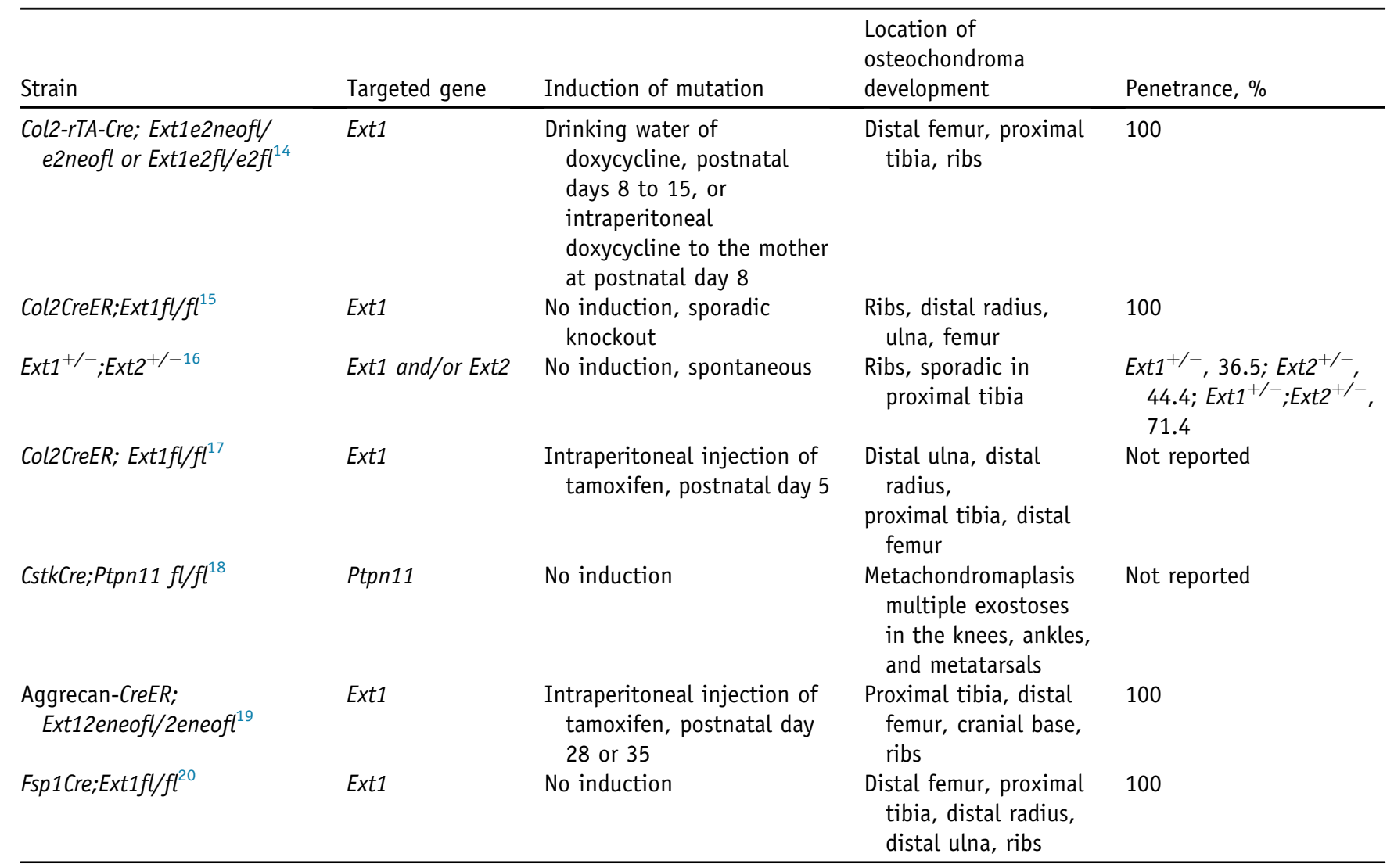

Osteochondromas arise with a $100 \%$ penetrance in long bones. Growth plate of the femur and tibia were stained with $\mathrm{X}$-gal and showed a low frequency of lac $\mathrm{Z}^{+}$cells that form clusters that correspond to the columnar organization of chondrocytes in the growth plate. No lac $\mathrm{Z}^{+}$cells were found in the perichondrium, including the area known as the groove of Ranvier, the perichondrium adjacent to growth plate. The study suggests that the cellular origin of osteochondromas is within the growth plate and that biallelic inactivation of Ext1 is important in the initiation of osteochondroma formation but might not be necessary for growing osteochondromas in mice.

\section{Ext1 $1^{+/-}$and Ext2 $^{+/-}$Heterozygotes}

Efforts to recapitulate the human HME genotypes that involve heterozygous mutations of the EXT1 and EXT2 allele have been investigated. Zak et al $^{16}$ have created compound $E x t 1^{+/-} ; E x t 2^{+/-}$heterozygote mice and compared the phenotype of either Ext1 and/or Ext2 null allele mice with double heterozygote mice by monitoring ectopic cartilage development located in the ribs and long bones. The $E x t 1^{+/-} ; E x t 2^{+/-}$heterozygote mice revealed $71.4 \%$ penetrance, whereas the single Ext1 and Ext2 heterozygote had $36.5 \%$ to $44.4 \%$ penetrance, respectively. In addition to rib outgrowths, the compound $E x t 1^{+/-} ; E x t 2^{+/-}$ mutants have exhibited osteochondromas in the tibia and femur. Histologic analysis has shown that the periosteal border is interrupted by the developing osteochondromas that consisted of growth plate-like arrangement of chondrocytes. This model might be suitable for the study on cellular and molecular mechanisms underlying initiation of osteochondromas because osteochondromas in other mouse models are mostly induced by deletion of both Ext1 alleles. It has also been demonstrated that $E x t 2^{+/-}$mice have formed multiple osteochondromas in ribs with an approximate $1: 3$ ratio and represented abnormality in endochondral ossification with $100 \%$ penetrance. $^{23}$

\section{Col2CreER;Ext1fl/fl with Tamoxifen}

The same Col2-CreERT;Ext1fl/fl system was used but was performed with tamoxifen induction. ${ }^{17}$ On tamoxifen injection at postnatal day 5, Col2-CreERT;Ext1fl/fl mice developed ectopic cartilaginous outgrowths on the outer region surrounding the mutant growth plate as early as 1 week after tamoxifen induction. The histologic findings of cartilage deformation were similar to other reports that mimic osteochondroma development. ${ }^{14,15}$ In addition, developmental delay of the secondary ossification center in long bones and abnormality of articular chondrocytes, including ectopic expression of collagen $\mathrm{X}$ and matrix metalloproteinase 13 and hypertrophy in shape, were 
observed. The changes on these diverse cell populations suggest additional function of Extl in chondrocytes.

\section{CtskCre;Ptpn11fl/fl}

Yang et $\mathrm{al}^{18}$ generated a mouse model with a conditional deletion of Ptpn11, a tyrosine phosphatase called SHP2 in the cells where cathepsin $\mathrm{K}$ promoter activity is activated, and demonstrated that this mouse represents metachondromatosis, characterized by the development of endochondromas and osteochondromas. Interestingly, most cells in the groove of Ranvier are affected in this mouse system and form chondromas. These findings indicate that the perichondrial cells in the groove of Ranvier can potentially be the origin of osteochondromas. However, growth plate chondrocytes should not be excluded as the source of osteochondroma because lineage studies trace some growth plate chondrocytes as descenders of cathepsin $\mathrm{K}$ lineage cells. $^{18}$

\section{AggrecanCreER;Ext1e2neofl/e2neofl with Tamoxifen}

Sinha et $\mathrm{al}^{19}$ generated the AggrecanCreER;Ext1e2neofl/ e2neofl osteochondroma model and observed osteochondroma-like outgrowths in the cranial base on tamoxifen injection. Formation of osteochondromas in the cranial base has been found in a number of HME patients. ${ }^{19} \mathrm{~A}$ combination with reporter mice has demonstrated that the responding cells are the bulk of synchondrosis growth plates and the cells located along the chondroperichondrial border, showing similar pathologic findings elicited by Col2CreER;Ext12neof//2neofl with and without the tamoxifen system. ${ }^{15,17}$ These mutant mice have developed osteochondromas in both long bones and ribs at $100 \%$ penetrance on tamoxifen induction. This study demonstrated that Smadmediated BMP activation is critical for osteochondroma formation in this model.

\section{Fsp1Cre;Ext1fl/fl}

Inubushi et $\mathrm{al}^{20}$ performed a perichondrium-targeted conditional knockout of Extl using fibroblast-specific protein 1 (Fsp1) that was expressed in the perichondrium. This model involves the deletion of the Extl gene in progenitor cells in the perichondrium that undergo aberrant differentiation into chondrocytes but not chondrocytes in the growth plate. Fsp1Cre;Extlfl/fl mice developed osteochondromas that are similar to those seen in cartilage-targeted conditional knockout of Ext1. ${ }^{14,15,17,19}$ Fsp1Cre;Extlflfl mutants not only develop multiple osteochondromas at $100 \%$ penetrance in the long bones and ribs but also grow normally without any growth retardation and survived for longer than 1 year. The observations seen in this mouse model indicate that cell origin of osteochondromas can be additional progenitor cells in the perichondrium and that inhibition of BMP signaling can effectively suppress osteochondroma formation.

\section{Signaling Pathways Involved in Osteochondromas}

Endochondral ossification is an essential process that creates bone from cartilaginous templates in appendicular and axial skeletons. Initially, skeletal progenitors condense and differentiate into chondrocytes in programed regions at embryonic stages. Cartilage development and endochondral ossification are controlled by an interplay of intricate signaling networks that involve the surrounding matrix, secreted morphogens, and growth factors, such as BMPs, Indian hedgehog (IHH), Wnts, and fibroblast growth factors. ${ }^{24,25}$ Studies have indicated that dysregulation of those signaling pathways can cause or are associated with pathology of osteochondroma formation. ${ }^{12,26}$

\section{BMP Signaling}

BMPs contain a group of growth factors. ${ }^{27,28}$ BMP signaling gradients exist across the growth plate and regulate bone formation and homeostasis, development, or morphogenesis of cartilage. BMPs bind to BMP receptor type I(BMPR-I) and BMP receptor type II (BMPR-II) transmembrane receptor proteins. Once this binding and receptor dimerization of BMPR-I and BMPR-II occurs, BMPR-II phosphorylates the GS domain of BMPR-I, causing the phosphorylation of signal-transduction proteins Smad 1, 5, or 8 (R-Smads) in the cell. ${ }^{28}$ Smad 6 and 7 (I-Smads) inhibit and modulate the phosphorylation of R-Smads. In addition, R-Smad interacts with Smad 4 (co-Smad) to form a complex that translocates to the nucleus and activates the transcription of BMP-responsive genes. ${ }^{27,28}$ More than 15 BMPs have been identified, and among them, BMP-2, BMP-4, and BMP-7 regulate the growth and maturation of chondrocytes in vitro, whereas BMP-2, BMP-4, BMP-6, and BMP-7 induce bone and cartilage formation in vivo. ${ }^{28}$ Dysregulation of the BMP signaling pathway is involved in the disease fibrodysplasia ossificans progressiva (FOP), which is a rare autosomal dominant disorder with mutations of ACVR1 that result in progressive heterotopic ossification of skeletal muscle and soft connective tissue. ${ }^{29}$ These findings indicate a close relation of BMP signaling with ectopic cartilage formation.

HSPGs are responsible for sequestering BMPs at the cell surface or in extracellular matrix while also enhancing BMP activity by continuously serving BMPs to their signaling receptors on the cell surface. ${ }^{30}$ Gene expression of BMP2 and BMPRIB and nuclear localization of phospho-Smad1/5/ 8 have been observed in the cartilage caps of human osteochondroma specimens, ${ }^{31}$ suggesting that enhanced BMP signaling might play a role in osteochondroma 
formation. Cellular pSmad1/5/8 levels have been increased in the perichondrium by Extl deficiency in organ culture and mouse osteochondroma model. ${ }^{20,32}$ Pharmacologic inhibition of BMP signaling by the LDN-193189 inhibitor reduced osteochondroma formation in osteochondroma mouse models. ${ }^{19,20}$ These findings support the idea that activation or dysregulation of BMP signaling in growth plate chondrocytes and/or perichondrium cells can underlie osteochondroma progression and formation.

\section{IHH Signaling}

The core components of the $\mathrm{HH}$ pathway is composed of three different $\mathrm{HH}$ ligands (Sonic HH, Desert HH, and IHH), a 12-pass transmembrane receptor patched1 (PTCH1), and a G-protein-coupled receptor-like sevenpass transmembrane protein smoothened (SMO). ${ }^{33,34}$ In the absence of HH ligand, PTCH1 sequesters SMO, inhibiting SMO activation. However, when $\mathrm{HH}$ is present, this repressive action is released. SMO forms a complex with the Ellis-van Creveld (EVC) syndrome protein and EVC2, triggering downstream trafficking and processing of GLI transcription factors. Three transcription factors (GLI1, GLI2, and GLI3) mediate HH signaling. ${ }^{34}$ During $\mathrm{HH}$ activation, decreasing cAMP levels lead to restriction of protein kinase A activity, allowing GLI2 and GLI3 to maintain their full length status and bypass phosphorylation, preventing its degradation and resulting in $\mathrm{HH}$ activation. ${ }^{33}$ This process allows active GLI $/ 3$ translocation to the nucleus and initiates the expression of $\mathrm{HH}$ target genes, such as GLI1 and PTCH1. GLI targets determine cell fate of tissue patterning, cell proliferation, and cell survival regulators. $^{33}$

HHs contain the HS/heparin binding domain that mediates interaction with HSPGs and include a consensus Cardin-Weintraub motif. ${ }^{30} \mathrm{IHH}$ is one of the main regulators of chondrocyte differentiation in the normal growth plate. It is produced by prehypertrophic chondrocytes and controls proliferation and the onset of hypertrophic differentiation of chondrocytes and osteoblast differentiation. ${ }^{24,35}$ The analysis of achondroplasia seen in brachymorphic mice have revealed that abnormal distribution of Ihh proteins and a decrease in Ihh signaling in the growth plate are associated with undersulfation of the chondroitin sulfate proteoglycans. ${ }^{36}$ This finding suggests that undersulfated HS might affect the diffusion of Ihh proteins and Ihh signaling.

Koziel et $\mathrm{al}^{37}$ found that HS negatively regulates the range of hedgehog signaling in a concentration-dependent manner in the growth plate and that reduced HS biosynthesis caused by hypomorphic mutation on Extl resulting in increased chondrocyte proliferation and delayed onset of hypertrophy via elevated Ihh signaling in embryonic bone formation in mice. Ext1 ${ }^{+/-}$mouse embryos have also represented an enhanced Ihh diffusion in the tibia with increased proliferation and delayed hypertrophy of growth plate chondrocytes. ${ }^{38}$ These findings lead to the theory that the development of osteochondromas might be attributed to the inability of HS to regulate the distribution of IHH. However, haploinsufficiency of Ihh does not change the development of osteochondromas as determined by tumor size and number, whereas $\mathrm{HH}$ signaling activation by deletion of an allele of Ptchl, reduces the number of tumors. ${ }^{39}$ The same study found that in a subset of osteochondromas, Ihh activation increased the cellularity and inhibited chondrocyte maturation, raising concern about whether changes in $\mathrm{HH}$ signaling activity might predispose to individuals to osteochondroma transformation. ${ }^{39}$ Of note, data from humans indicate that increased IHH activation is linked to chondrosarcoma development, ${ }^{40}$ even though the study found that a decrease in IHH signaling is associated with malignant transformation toward secondary peripheral chondrosarcoma in human specimens. ${ }^{41}$ Evidence that supports the role of IHH signaling in osteochondromas is still limited and should be further studied.

\section{Wnt/ $\beta$-Catenin Signaling}

The Wnt family is a group of highly conserved proteins responsible for cell development and homeostasis. ${ }^{42}$ The Wnt pathway includes 19 Wnt molecules and 15 frizzled receptors and co-receptors, such as low density lipoprotein receptor-related protein cell surface markers. ${ }^{42}$ In addition, the pathway is also regulated by extracellular and intercellular inhibitors, including secreted Frizzled-related proteins, Wnt inhibitor factor-1, Dickkopfs, and axis inhibition proteins). ${ }^{42} \mathrm{Wnt} / \beta$-catenin signaling is a major signal transduction pathway of Wnts. Once Wnt proteins bind to the cell surface receptor complexes Frizzleds and LRP5/6, downstream intercellular protein Disheveled is activated, resulting in the inhibition of glycogen synthase- $3 \beta$ kinase and $\beta$-catenin phosphorylation. ${ }^{42}$ This inhibition then allows $\beta$-catenin to escape the ubiquitin proteasome degradation pathway, allowing $\beta$-catenin accumulation in the cytoplasm. ${ }^{42}$ Stabilized nonphosphorylated $\beta$-catenin is then able to translocate to the nucleus and interacts with multiple transcription factors, activating transcription of Wnttargeted genes. Wnt/ $\beta$-catenin signaling also strongly influences development and organization of growth plates. Research on the roles of this signaling pathway in limb development and growth have been performed using various transgenic mice. The findings support that proper control of the signaling activity is essential for joint formation, growth plate function, and skeletal formation. ${ }^{43}$

Wnt/ $\beta$-catenin signaling is also modulated by HS, like HH signaling. ${ }^{44}$ HSPGs have been implicated in regulating the distribution and receptor binding of several members of Wnt at the cell surface and thereby support the solubility of hydrophobic Wnt and its activity. ${ }^{44}$ The importance of HS proteoglycans to regulate Wnt activity has been initially addressed in Drosophila and later in vertebrate models. ${ }^{44}$ In general, in a variety of cancers, Wnt/ $\beta$-catenin 
pathways are known to be upregulated, causing part of the pathogenesis, but this scenario might not be the same for osteochondroma pathogenesis. Interestingly, $\beta$-catenin conditional deletion induces ectopic chondroma-like cartilage formation in mice during cartilage development. ${ }^{45}$ When an $\beta$-catenin signaling stimulator is present, apoptotic and cell death levels are elevated in cultured wildtype chondrocytes. In addition, immunohistochemical analysis of hereditary multiple exostoses specimens revealed that the amount of detectable $\beta$-catenin in cartilage cells of osteochondromas was much lower than that in hypertrophic chondrocytes in normal human growth plates. ${ }^{45}$ These findings suggest that inhibition of Wnt- $\beta$-catenin signaling is related to the development of osteochondroma formation. Moreover, $\mathrm{Wnt} / \beta$-catenin signaling was found to be an essential fate determinant of mesenchymal cells to osteoblasts or chondrocytes and strongly suggests involvement of this signaling in the pathogenesis of osteochondroma. Wnt/ $\beta$-catenin signaling activation regulates both cartilage development and homeostasis and shows diverse effects on chondrocyte differentiation, proliferation, and survival. ${ }^{43}$ This finding might explain why both genetic stimulation and reduction of $\mathrm{Wnt} / \beta$-catenin signaling suppressed osteochondroma formation in mice. ${ }^{39}$ Future studies are needed to evaluate the mechanism behind $\mathrm{Wnt} / \beta$-catenin signaling in the reduction of osteochondroma formation.

\section{Interaction of RAR- $\gamma$ Signaling with Signaling Pathways in 0steochondroma Pathogenesis}

\section{Retinoid Signaling and Endochondral Ossification}

Retinols enter the cell through retinol binding protein receptor (stimulated by retinoic acid 6). Retinol is bound to cellular retinol binding protein and is converted into retinaldehyde by retinol dehydrogenase, which is then subsequently converted into retinoic acid by retinaldehyde dehydrogenase. ${ }^{46}$ Retinoic acids exert their functions

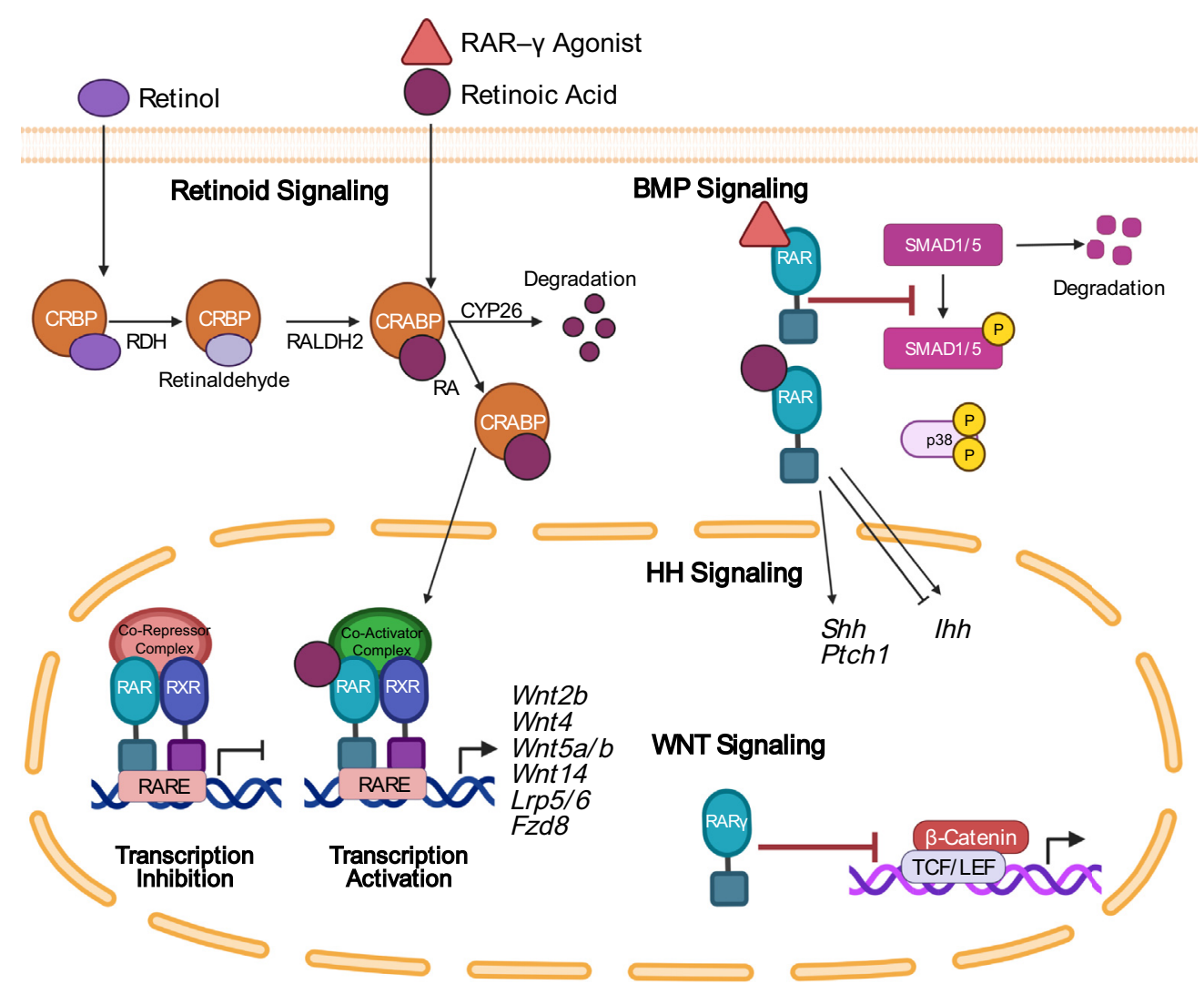

Figure 1 Possible interactions of the retinoid pathway with bone morphogenetic protein, Hedgehog $(\mathrm{HH})$, and WNT/ $\beta$-catenin signaling. Retinol enters the cytoplasm and is converted into an active form: retinoic acid (RA). Cellular retinoic acid-binding protein (CRABP)-bound RA is translocated to the nucleus. On the nuclear binding of RA to retinoic acid receptors (RARs), conformational changes cause the ligand-binding domain to expose a binding site for coactivators. RARs and retinoic $X$ receptors (RXRs) form heterodimeric complexes and bind to retinoic acid response elements (RAREs) located at the enhancer regions of RA target genes and initiate gene transcription. In the absence of ligands, the coactivators of RARs and RXRs are replaced by co-repressors, resulting in transcriptional repression of the target genes. RAR agonists inhibit phosphorylation of Smad1/5 and stimulate degradation of Smad1/5. RA stimulates gene expression of Shh and Ptch1 in chondrocytes and regulates Ihh gene expression negatively and positively in chondrocytes. RA stimulates gene expression of Wnt2b, Wnt4, Wnt5a, Wnt5b, Wnt14, Fzd8, and Lrp5 and 6 in mouse chondrocytes. Unliganded RAR- $\gamma$ is associated with $\beta$-catenin and inhibits the $\beta$-catenin transcriptional activity in mouse chondrocytes. The figure was created with BioRender.com (BioRender, Toronto, ON, Canada). 
through two specific classes of receptors: RARs and retinoic X receptors (RXRs). Each class contains three subtypes: $\alpha, \beta$, and $\gamma \cdot{ }^{47}$ The expression of these receptors is regulated by the receptors themselves, by nuclear receptors, or by other subtypes in the same family. ${ }^{48}$ On the nuclear binding of retinoic acid to RARs, conformational changes cause the ligand-binding domain to expose a binding site for coactivators, including $\mathrm{CBP} / \mathrm{p} 300$, p300/ CBP-associated factor, and p160 family members, which then recruit proteins that contain histone acetyltransferase activity. ${ }^{49}$ RARs and RXRs form heterodimeric complexes to function as ligand-dependent transcription factors and bind to retinoic acid response elements located at the enhancer regions of target genes. Unliganded receptors are responsible for repressing target gene expression. ${ }^{50}$ In the absence of ligands, the coactivators of RARs/RXRs are replaced by co-repressors, which include nuclear receptor c-repressor 1/2 (, silencing mediators of RARs and thyroid hormone receptors), $\mathrm{mSin} 3 \mathrm{~A}$, and histone deacetylases. Together this complex results in transcriptional repression of the target genes of RAR/RXR heterodimers ${ }^{50,51}$ (Figure 1).

Retinoic acid is a negative regulator of growth plate chondrogenesis and can be influenced by the expression and/or the activity of SOX9, a major chondrogenic driver. ${ }^{52}$ Retinoid signaling strongly inhibits growth plate chondrocyte proliferation as shown in the retinoic acid-treated metatarsal organ culture ${ }^{53}$ and in Cyp26b1-deficient mice ${ }^{54}$ Furthermore, retinoic acid suppresses cartilage matrix synthesis in growth plate chondrocyte cultures. ${ }^{55,56} \mathrm{In}$ cephalic chick chondrocyte cultures, retinoic acid stimulates chondrocyte hypertrophy and mineralization. ${ }^{57}$ Genes encoding RAR- $\alpha$, RAR- $\beta$, and RAR- $\gamma$ display spatiotemporal patterns of expression during cartilage formation. RAR- $\alpha$ is expressed throughout the limb mesenchyme early in limb development, decreases as cells begin to differentiate into chondrocytes, and then remains low in cartilaginous tissues. ${ }^{58}$ RAR- $\beta$ is expressed in regions of the developing limb that are not destined to form cartilage, whereas RAR- $\gamma$ is expressed preferentially in both prechondrogenic mesenchymal condensations and newly differentiated chondrocytes before chondrocytes hypertrophy, with respect to skeletal development in the limb. ${ }^{59-61}$ In limb bud cells and chondrocytes, RAR- $\gamma-$ mediated retinoic acid action strongly inhibits chondrogenesis and cartilage matrix synthesis.,

Synthetic retinoid agonists specific for RAR- $\gamma$, such as palovarotene, function as potent inhibitors of osteochondromas in rodent models. ${ }^{8}$ The RAR- $\gamma$ agonist can exert antitumor function on human osteochondromas by inhibiting matrix synthesis, promoting cartilage matrix degradation, and stimulating cell death. ${ }^{62}$ The inhibitory action of RAR- $\gamma$ signaling on osteochondromas might be mediated by interaction of RAR- $\gamma$ signaling with the multiple signaling pathways that are important for cartilage development and endochondral ossification.
Interaction of Retinoid Signaling with $\mathrm{BMP}, \mathrm{HH}$, and Wnt/ $\beta$-Catenin Signaling Pathways

Heterotopic ossification involves induction of ectopic endochondral ossification and can be triggered by uncontrolled activation of BMP signaling, as observed in diseases such as FOP, which are associated with genetic mutations in BMP type I receptor ACVR1. ${ }^{29}$ RAR- $\gamma$ agonists inhibit phosphorylation of Smad1/5 and stimulate degradation of Smad1/5 proteins. ${ }^{5}$ BMP inhibition can be an attribute to chondrogenic inhibition by RAR- $\gamma$ agonists, which typically requires a decrease in retinoid signaling while inversely upregulating BMP signaling. ${ }^{63}$ A similar inhibition of Smad1/5/8 phosphorylation by the RAR- $\gamma$ agonists is addressed in the mouse osteochondroma model. ${ }^{8}$ In addition, pharmacologic interference of RAR- $\gamma$ stimulates Smad1/5 phosphorylation. ${ }^{64}$ Therefore, it is very likely that inhibition of BMP signaling is an important mechanism in which RAR- $\gamma$ agonists suppress ectopic cartilage formation in osteochondromas (Figure 1).

RAR- $\gamma$ transcripts are detected in prehypertrophic chondrocytes, overlapping with $I h h$ gene expression in E18 mandibular condyle. Shimo et $\mathrm{al}^{65}$ studied the crossinteraction between retinoid and Ihh signaling pathways using chick cephalic chondrocyte culture system. The authors found that retinoic acid stimulates Ihh gene expression, which requires de novo protein synthesis and activation of p38 mitogen-activated protein kinase, which is important for retinoic acid-induced $I h h$ gene expression, and that retinoic acid inhibits proliferation of proliferating chondrocytes but not that of hypertrophic chondrocytes. ${ }^{65}$ Yoshida et $\mathrm{al}^{66}$ found that the $5^{\prime}$ flanking region of the human $\mathrm{IHH}$ gene contains a retinoic acid response element and that retinoic acid treatment upregulates $I h h$ and Ptchedl gene expression in high-density cultures of rabbit growth-plate chondrocytes. Conversely, $\mathrm{Wu}$ et $\mathrm{al}^{67} \mathrm{re}-$ ported that Shh and Ihh are expressed in growth plate by quantitative PCR and in situ hybridization and that retinoic acid stimulates gene expression of Shh but inhibits Ihh expression. These findings suggest that retinoid signaling can alter $\mathrm{HH}$ signaling in both positive and negative ways (Figure 1). Considering the finding that Ihh potentiates BMP signaling and induces ectopic cartilage formation, the RAR- $\gamma$ agonist might inhibit HH signaling when it inhibits ectopic cartilage formation and osteochondromas, but the interaction between retinoid and $\mathrm{HH}$ signaling in osteochondromas can vary, depending on the stage of tumor development and the microenvironment of where and how tumors grow.

Wnt/ $\beta$-catenin signaling molecules interact with the nuclear receptor family members in diverse types of cells, tissues, and organs and are developmentally and clinically important. ${ }^{68}$ Cross-talking among Wnt, retinoid, HH, BMP, and fibroblast growth factor signaling pathways sophisticatedly regulates limb morphogenesis. ${ }^{69}$ Spatiotemporal expression of the ligands, receptors, and regulatory 
molecules of these signaling pathways coordinates signaling gradients and specifies the anterior-posterior axis and the proximo-distal pattern. ${ }^{69}$ Retinoic acid plays a role in specification of the proximal part of the limb, Wnt3 and Wnt5a contribute to the distal growth, and Wnt7a contributes to the ventralization. ${ }^{69,70}$ However, the molecular interaction between retinoid and $\mathrm{Wnt} / \beta$-catenin signaling during limb morphogenesis is not yet elucidated.

Retinoic acid treatment increases gene expression of the Wnts (Wnt2b, Wnt4, Wnt5a, Wnt5b, and Wnt14) and receptors and coreceptors (Fzd8, Lrp5, and Lrp6) and enhances $\mathrm{Wnt} / \beta$-catenin signaling. ${ }^{71}$ In conditions where retinoid ligands are not available in normal chondrocytes, unliganded RAR- $\gamma$ is associated with $\beta$-catenin via the amino-terminal domain of RAR- $\gamma$ and inhibits the $\beta$-catenin transcriptional activity with lymphoid enhancer factor/T-cell factor transcription factors. ${ }^{71}$ Therefore, alterations in RAR- $\gamma$ signaling can change Wnt/ $\beta$-catenin activity in chondrogenesis, endochondral ossification, and possibly in osteochondromas. Wnt/ $\beta$-catenin signaling can be stimulated by treatment with the RAR- $\gamma$ agonists, leading to inhibition of growth of osteochondromas (Figure 1).

\section{Palovarotene}

Two clinical trials of palovarotene were conducted as potential treatments for FOP (https://clinicaltrials.gov, trial number NCT03312634) and for multiple osteochondromas (https:// clinicaltrials.gov, trial number NCT03442985). The US Food and Drug Administration placed both trials on hold in response to cases of early plate closure in FOP pediatric patients. The multiple osteochondromas trial was later terminated (Ipsen Pharma, https://www.ipsen.com/websites/ Ipsen_Online/wp-content/uploads/2020/03/09112219/IpsenPalovarotene-Update-26-March-2020.pdf, last accessed July 14, 2021), and the FOP study was refined to enroll children 14 years and older and resumed only for those patients $>14$ years of age.

The multiple osteochondromas clinical trial evaluated two daily dosage regimens $(2.5$ and $5.0 \mathrm{mg})$ in 194 pediatric patients. The FOP clinical trial evaluated different dosage regimens of 5,10 , or $20 \mathrm{mg}$ in 54 pediatric patients. It has not been concluded which doses of palovarotene cause disorders of growth plate in pediatric patients. The negative effect of palovarotene treatment on skeletal tissues has also been observed in preclinical studies. ${ }^{8,72}$ When palovarotene is administered daily via intraperitoneal injections in mice from postnatal day 14 , the treatment disrupts growth plate and synovial joint morphology in juvenile FOP mice. Palovarotene-treated wild-type and FOP mice had growth plate loss and shortened body lengths, spine lengths, and tibial lengths compared with untreated control mice. ${ }^{72}$ Inubushi et $\mathrm{al}^{8}$ also detected inhibition of skeletal growth with oral gavage of palovarotene starting from postnatal day 14 , but deformity was not detected when treatment was initiated from postnatal day 21 with the same dose of palovarotene. The findings from the animal experiments suggest that susceptibility of the growth plate to palovarotene might reduce at older ages and that the route of palovarotene administration seems to be an important risk for skeletal deformity. Additional studies are required to evaluate the skeletal toxicity, including growth inhibition and joint degeneration, to determine the therapeutic potential of palovarotene.

\section{Conclusions}

Local application of RAR- $\gamma$ agonists abolished growth of transplanted human chondrosarcoma cells in a xenograft mouse model, ${ }^{62}$ suggesting that RAR- $\gamma$ agonists can be locally applied. A local treatment would be an alternative option of RAR- $\gamma$ agonist treatment, which might lower the risk of the drug adverse effects but should be investigated intensively. Currently, the clinical approach is a careful follow-up for small lamps of osteochondromas until the masses grow to meet surgery application criteria. Drug therapy delivery, however, might effectively inhibit tumor progression if applied at the beginning stage when osteochondromas are small masses.

Palovarotene, an RAR- $\gamma$ selective agonist, was originally investigated as a possible drug for emphysema and is currently proposed as a potent inhibitor drug for FOP, suggesting potential therapeutic benefits in various types of heterotopic ossification. ${ }^{5}$ Pharmacologic activation of RAR- $\gamma$ signaling must alter various signaling pathways in cartilage tissues (articular cartilage and growth plate) as described here. Understanding the interaction of RAR- $\gamma$ signaling with these important signaling pathways is vital to elucidate not only the pharmacologic action of RAR- $\gamma$ agonist drugs on heterotopic ossification and osteochondromas but also the physiologic roles of RAR- $\gamma-$ mediated retinoid signaling in endochondral ossification.

\section{References}

1. Pannier S, Legeai-Mallet L: Hereditary multiple exostoses and enchondromatosis. Best Pract Res Clin Rheumatol 2008, 22:45-54

2. de Souza AM, Bispo Junior RZ: Osteochondroma: ignore or investigate? Rev Bras Ortop 2014, 49:555-564

3. Chhina H, Davis JC, Alvarez CM: Health-related quality of life in people with hereditary multiple exostoses. J Pediatr Orthop 2012, 32: $210-214$

4. Williams JA, Kondo N, Okabe T, Takeshita N, Pilchak DM, Koyama E, Ochiai T, Jensen D, Chu ML, Kane MA, Napoli JL, Enomoto-Iwamoto M, Ghyselinck N, Chambon P, Pacifici M, Iwamoto M: Retinoic acid receptors are required for skeletal growth, matrix homeostasis and growth plate function in postnatal mouse. Dev Biol 2009, 328:315-327

5. Shimono K, Tung WE, Macolino C, Chi AH, Didizian JH, Mundy C, Chandraratna RA, Mishina Y, Enomoto-Iwamoto M, Pacifici M, Iwamoto M: Potent inhibition of heterotopic ossification by nuclear retinoic acid receptor-gamma agonists. Nat Med 2011, 17:454-460 
6. Chakkalakal SA, Uchibe K, Convente MR, Zhang D, Economides AN, Kaplan FS, Pacifici M, Iwamoto M, Shore EM: Palovarotene inhibits heterotopic ossification and maintains limb mobility and growth in mice with the human ACVR1(R206H) fibrodysplasia ossificans progressiva (FOP) mutation. J Bone Miner Res 2016, 31:1666-1675

7. Wheatley BM, Cilwa KE, Dey D, Qureshi AT, Seavey JG, Tomasino AM, Sanders EM, Bova W, Boehm CA, Iwamoto M, Potter BK, Forsberg JA, Muschler GF, Davis TA: Palovarotene inhibits connective tissue progenitor cell proliferation in a rat model of combat-related heterotopic ossification. J Orthop Res 2018, 36: $1135-1144$

8. Inubushi T, Lemire I, Irie F, Yamaguchi Y: Palovarotene inhibits osteochondroma formation in a mouse model of multiple hereditary exostoses. J Bone Miner Res 2018, 33:658-666

9. Ahn J, Ludecke HJ, Lindow S, Horton WA, Lee B, Wagner MJ, Horsthemke B, Wells DE: Cloning of the putative tumour suppressor gene for hereditary multiple exostoses (EXT1). Nat Genet 1995, 11: 137-143

10. Stickens D, Clines G, Burbee D, Ramos P, Thomas S, Hogue D, Hecht JT, Lovett M, Evans GA: The EXT2 multiple exostoses gene defines a family of putative tumour suppressor genes. Nat Genet 1996, 14:25-32

11. Hameetman L, Bovee JV, Taminiau AH, Kroon HM, Hogendoorn PC: Multiple osteochondromas: clinicopathological and genetic spectrum and suggestions for clinical management. Hered Cancer Clin Pract 2004, 2:161-173

12. Zak BM, Crawford BE, Esko JD: Hereditary multiple exostoses and heparan sulfate polymerization. Biochim Biophys Acta 2002, 1573: 346-355

13. Poulain FE, Yost HJ: Heparan sulfate proteoglycans: a sugar code for vertebrate development? Development 2015, 142:3456-3467

14. Jones KB, Piombo V, Searby C, Kurriger G, Yang B, Grabellus F, Roughley PJ, Morcuende JA, Buckwalter JA, Capecchi MR, Vortkamp A, Sheffield VC: A mouse model of osteochondromagenesis from clonal inactivation of Ext1 in chondrocytes. Proc Natl Acad Sci U S A 2010, 107:2054-2059

15. Matsumoto K, Irie F, Mackem S, Yamaguchi Y: A mouse model of chondrocyte-specific somatic mutation reveals a role for Ext1 loss of heterozygosity in multiple hereditary exostoses. Proc Natl Acad Sci U S A 2010, 107:10932-10937

16. Zak BM, Schuksz M, Koyama E, Mundy C, Wells DE, Yamaguchi Y, Pacifici M, Esko JD: Compound heterozygous loss of Ext1 and Ext2 is sufficient for formation of multiple exostoses in mouse ribs and long bones. Bone 2011, 48:979-987

17. Sgariglia F, Candela ME, Huegel J, Jacenko O, Koyama E, Yamaguchi Y, Pacifici M, Enomoto-Iwamoto M: Epiphyseal abnormalities, trabecular bone loss and articular chondrocyte hypertrophy develop in the long bones of postnatal Ext1-deficient mice. Bone 2013, 57:220-231

18. Yang W, Wang J, Moore DC, Liang H, Dooner M, Wu Q, Terek R, Chen Q, Ehrlich MG, Quesenberry PJ, Neel BG: Ptpn11 deletion in a novel progenitor causes metachondromatosis by inducing hedgehog signalling. Nature 2013, 499:491-495

19. Sinha S, Mundy C, Bechtold T, Sgariglia F, Ibrahim MM, Billings PC, Carroll K, Koyama E, Jones KB, Pacifici M: Unsuspected osteochondroma-like outgrowths in the cranial base of hereditary multiple exostoses patients and modeling and treatment with a BMP antagonist in mice. PLoS Genet 2017, 13:e1006742

20. Inubushi T, Nozawa S, Matsumoto K, Irie F, Yamaguchi Y: Aberrant perichondrial BMP signaling mediates multiple osteochondromagenesis in mice. JCI Insight 2017, 2:e90049

21. Reijnders CM, Waaijer CJ, Hamilton A, Buddingh EP, Dijkstra SP, Ham J, Bakker E, Szuhai K, Karperien M, Hogendoorn PC, Stringer SE, Bovee JV: No haploinsufficiency but loss of heterozygosity for EXT in multiple osteochondromas. Am J Pathol 2010, 177: 1946-1957
22. Bovee JV, Cleton-Jansen AM, Wuyts W, Caethoven G, Taminiau AH, Bakker E, Van Hul W, Cornelisse CJ, Hogendoorn PC: EXT-mutation analysis and loss of heterozygosity in sporadic and hereditary osteochondromas and secondary chondrosarcomas. Am J Hum Genet 1999, 65:689-698

23. Stickens D, Zak BM, Rougier N, Esko JD, Werb Z: Mice deficient in Ext2 lack heparan sulfate and develop exostoses. Development 2005, 132:5055-5068

24. Long F, Ornitz DM: Development of the endochondral skeleton. Cold Spring Harb Perspect Biol 2013, 5:a008334

25. Ballock RT, O'Keefe RJ: Physiology and pathophysiology of the growth plate. Birth Defects Res C Embryo Today 2003, 69:123-143

26. Huegel J, Sgariglia F, Enomoto-Iwamoto M, Koyama E, Dormans JP, Pacifici M: Heparan sulfate in skeletal development, growth, and pathology: the case of hereditary multiple exostoses. Dev Dyn 2013, 242:1021-1032

27. Cuellar A, Reddi AH: Cell biology of osteochondromas: bone morphogenic protein signalling and heparan sulphates. Int Orthop 2013, 37:1591-1596

28. Pogue R, Lyons K: BMP signaling in the cartilage growth plate. Curr Top Dev Biol 2006, 76:1-48

29. Kaplan FS, Xu M, Seemann P, Connor JM, Glaser DL, Carroll L, Delai P, Fastnacht-Urban E, Forman SJ, Gillessen-Kaesbach G, Hoover-Fong J, Koster B, Pauli RM, Reardon W, Zaidi SA, Zasloff M, Morhart R, Mundlos S, Groppe J, Shore EM: Classic and atypical fibrodysplasia ossificans progressiva (FOP) phenotypes are caused by mutations in the bone morphogenetic protein (BMP) type I receptor ACVR1. Hum Mutat 2009, 30:379-390

30. Billings PC, Yang E, Mundy C, Pacifici M: Domains with highest heparan sulfate-binding affinity reside at opposite ends in BMP2/4 versus BMP5/6/7: implications for function. J Biol Chem 2018, 293: $14371-14383$

31. Nakase T, Myoui A, Shimada K, Kuriyama K, Joyama S, Miyaji T, Tomita T, Yoshikawa H: Involvement of BMP-2 signaling in a cartilage cap in osteochondroma. J Orthop Res 2001, 19:1085-1088

32. Huegel J, Mundy C, Sgariglia F, Nygren P, Billings PC, Yamaguchi Y, Koyama E, Pacifici M: Perichondrium phenotype and border function are regulated by Ext1 and heparan sulfate in developing long bones: a mechanism likely deranged in Hereditary Multiple Exostoses. Dev Biol 2013, 377:100-112

33. Ramsbottom SA, Pownall ME: Regulation of hedgehog signalling inside and outside the cell. J Dev Biol 2016, 4:23

34. Ingham PW, McMahon AP: Hedgehog signaling in animal development: paradigms and principles. Genes Dev 2001, 15:3059-3087

35. Vortkamp A, Lee K, Lanske B, Segre GV, Kronenberg HM, Tabin CJ: Regulation of rate of cartilage differentiation by Indian hedgehog and PTH-related protein. Science 1996, 273:613-622

36. Cortes M, Baria AT, Schwartz NB: Sulfation of chondroitin sulfate proteoglycans is necessary for proper Indian hedgehog signaling in the developing growth plate. Development 2009 , 136:1697-1706

37. Koziel L, Kunath M, Kelly OG, Vortkamp A: Ext1-dependent heparan sulfate regulates the range of Ihh signaling during endochondral ossification. Dev Cell 2004, 6:801-813

38. Hilton MJ, Gutierrez L, Martinez DA, Wells DE: EXT1 regulates chondrocyte proliferation and differentiation during endochondral bone development. Bone 2005, 36:379-386

39. Piombo V, Jochmann K, Hoffmann D, Wuelling M, Vortkamp A: Signaling systems affecting the severity of multiple osteochondromas. Bone 2018, 111:71-81

40. Bovee JV, Hogendoorn PC, Wunder JS, Alman BA: Cartilage tumours and bone development: molecular pathology and possible therapeutic targets. Nat Rev Cancer 2010, 10:481-488

41. Hameetman L, Rozeman LB, Lombaerts M, Oosting J, Taminiau AH, Cleton-Jansen AM, Bovee JV, Hogendoorn PC: Peripheral chondrosarcoma progression is accompanied by decreased Indian Hedgehog signalling. J Pathol 2006, 209:501-511 
42. Nusse R, Clevers $H$ : Wnt/beta-catenin signaling, disease, and emerging therapeutic modalities. Cell 2017, 169:985-999

43. Usami Y, Gunawardena AT, Iwamoto M, Enomoto-Iwamoto M: Wnt signaling in cartilage development and diseases: lessons from animal studies. Lab Invest 2016, 96:186-196

44. Xie M, Li JP: Heparan sulfate proteoglycan: a common receptor for diverse cytokines. Cell Signal 2019, 54:115-121

45. Cantley L, Saunders C, Guttenberg M, Candela ME, Ohta Y, Yasuhara R, Kondo N, Sgariglia F, Asai S, Zhang X, Qin L, Hecht JT, Chen D, Yamamoto M, Toyosawa S, Dormans JP, Esko JD, Yamaguchi $\mathrm{Y}$, Iwamoto M, Pacifici M, EnomotoIwamoto M: Loss of beta-catenin induces multifocal periosteal chondroma-like masses in mice. Am J Pathol 2013, 182:917-927

46. Green AC, Martin TJ, Purton LE: The role of vitamin A and retinoic acid receptor signaling in post-natal maintenance of bone. J Steroid Biochem Mol Biol 2016, 155:135-146

47. Alvarez S, Germain P, Alvarez R, Rodriguez-Barrios F, Gronemeyer H, de Lera AR: Structure, function and modulation of retinoic acid receptor beta, a tumor suppressor. Int J Biochem Cell Biol 2007, 39:1406-1415

48. Bushue N, Wan YJ: Retinoid pathway and cancer therapeutics. Adv Drug Deliv Rev 2010, 62:1285-1298

49. Glass CK, Rosenfeld MG: The coregulator exchange in transcriptional functions of nuclear receptors. Genes Dev 2000, 14:121-141

50. Weston AD, Blumberg B, Underhill TM: Active repression by unliganded retinoid receptors in development: less is sometimes more. J Cell Biol 2003, 161:223-228

51. Bastien J, Rochette-Egly C: Nuclear retinoid receptors and the transcription of retinoid-target genes. Gene 2004, 328:1-16

52. Weston AD, Rosen V, Chandraratna RA, Underhill TM: Regulation of skeletal progenitor differentiation by the BMP and retinoid signaling pathways. J Cell Biol 2000, 148:679-690

53. De Luca F, Uyeda JA, Mericq V, Mancilla EE, Yanovski JA, Barnes KM, Zile MH, Baron J: Retinoic acid is a potent regulator of growth plate chondrogenesis. Endocrinology 2000, 141: 346-353

54. Minegishi Y, Sakai Y, Yahara Y, Akiyama H, Yoshikawa H, Hosokawa K, Tsumaki N: Cyp26b1 within the growth plate regulates bone growth in juvenile mice. Biochem Biophys Res Commun 2014, 454:12-18

55. Hiraki Y, Yutani Y, Fukuya M, Takigawa M, Suzuki F: Differentiation and de-differentiation of cultured chondrocytes: increase in monomeric size of 'cartilage-specific' proteoglycans by dibutyryl cyclic AMP and complete inhibition of their synthesis by retinoic acid. Biochem Int 1985, 10:267-272

56. Iwamoto M, Kitagaki J, Tamamura Y, Gentili C, Koyama E, Enomoto H, Komori T, Pacifici M, Enomoto-Iwamoto M: Runx2 expression and action in chondrocytes are regulated by retinoid signaling and parathyroid hormone-related peptide (PTHrP). Osteoarthritis Cartilage 2003, 11:6-15

57. Iwamoto M, Yagami K, Shapiro IM, Leboy PS, Adams SL, Pacifici M: Retinoic acid is a major regulator of chondrocyte maturation and matrix mineralization. Microsc Res Tech 1994, 28:483-491
58. Weston AD, Chandraratna RA, Torchia J, Underhill TM: Requirement for RAR-mediated gene repression in skeletal progenitor differentiation. J Cell Biol 2002, 158:39-51

59. Dolle P, Ruberte E, Kastner P, Petkovich M, Stoner CM, Gudas LJ, Chambon P: Differential expression of genes encoding alpha, beta and gamma retinoic acid receptors and CRABP in the developing limbs of the mouse. Nature 1989, 342:702-705

60. Koyama E, Golden EB, Kirsch T, Adams SL, Chandraratna RA, Michaille JJ, Pacifici M: Retinoid signaling is required for chondrocyte maturation and endochondral bone formation during limb skeletogenesis. Dev Biol 1999, 208:375-391

61. Weston AD, Hoffman LM, Underhill TM: Revisiting the role of retinoid signaling in skeletal development. Birth Defects Res C Embryo Today 2003, 69:156-173

62. Garcia SA, Tian H, Imamura-Kawasawa Y, Fisher A, Cellini A, Codd C, Herzenberg JE, Abzug JM, Ng V, Iwamoto M, EnomotoIwamoto M: Understanding the action of RARgamma agonists on human osteochondroma explants. Int J Mol Sci 2020, 21:2686

63. Hoffman LM, Garcha K, Karamboulas K, Cowan MF, Drysdale LM, Horton WA, Underhill TM: BMP action in skeletogenesis involves attenuation of retinoid signaling. J Cell Biol 2006, 174:101-113

64. Uchibe K, Son J, Larmour C, Pacifici M, Enomoto-Iwamoto M, Iwamoto M: Genetic and pharmacological inhibition of retinoic acid receptor gamma function promotes endochondral bone formation. J Orthop Res 2017, 35:1096-1105

65. Shimo T, Koyama E, Ibaragi S, Naito K, Yamamoto D, Okui T, Kishimoto K, Mese H, Sasaki A: Possible involvement of p38 MAP kinase in retinoid stimulated expression of Indian hedgehog in prehypertrophic chondrocytes. Oral Science Int 2008, 5:1-14

66. Yoshida E, Noshiro M, Kawamoto T, Tsutsumi S, Kuruta Y, Kato Y: Direct inhibition of Indian hedgehog expression by parathyroid hormone (PTH)/PTH-related peptide and up-regulation by retinoic acid in growth plate chondrocyte cultures. Exp Cell Res 2001, 265:64-72

67. Wu LN, Lu M, Genge BR, Guo GY, Nie D, Wuthier RE: Discovery of sonic hedgehog expression in postnatal growth plate chondrocytes: differential regulation of sonic and Indian hedgehog by retinoic acid. J Cell Biochem 2002, 87:173-187

68. Mulholland DJ, Dedhar S, Coetzee GA, Nelson CC: Interaction of nuclear receptors with the Wnt/beta-catenin/Tcf signaling axis: wnt you like to know? Endocr Rev 2005, 26:898-915

69. Towers M, Wolpert L, Tickle C: Gradients of signalling in the developing limb. Curr Opin Cell Biol 2012, 24:181-187

70. Yang Y: Wnts and wing: wnt signaling in vertebrate limb development and musculoskeletal morphogenesis. Birth Defects Res C Embryo Today 2003, 69:305-317

71. Yasuhara R, Yuasa T, Williams JA, Byers SW, Shah S, Pacifici M, Iwamoto M, Enomoto-Iwamoto M: Wnt/beta-catenin and retinoic acid receptor signaling pathways interact to regulate chondrocyte function and matrix turnover. J Biol Chem 2010, 285:317-327

72. Lees-Shepard JB, Nicholas SE, Stoessel SJ, Devarakonda PM, Schneider MJ, Yamamoto M, Goldhamer DJ: Palovarotene reduces heterotopic ossification in juvenile FOP mice but exhibits pronounced skeletal toxicity. Elife 2018, 7:e40814 